\title{
URINARY TRACT INFECTION: DO WE FOLLOW EVM? CURRENT GUIDELINES
}

\author{
MA SALAM ${ }^{1}$, IBRAHIM KAISER ${ }^{2}$, F ISLAM $^{3}$, J KABIR ${ }^{4}$, SA KHAN ${ }^{5}$, SAHA P ${ }^{6}$
}

\section{Purpose}

The purpose of this guideline is to provide up-to-date information for diagnosis and treatment of asymptomatic bacteriuria in adult. These recommendations were developed on the basis of a review of published evidence, with the strength of the recommendation described by Infectious Diseases Society of America (IDSA)[1]. This guideline is not meant to replace clinical judgment.

\section{Definitions}

Asymptomatic bacteriuria," or asymptomatic urinary infection, is isolation of a specified quantitative count of bacteria in an appropriately collected urine specimen obtained from a person without symptoms or signs referable to urinary infection [1].

Acute uncomplicated urinary tract infection is a symptomatic bladder infection characterized by frequency, urgency, dysuria, or suprapubic pain in a woman with a normal genitourinary tract, and it is associated with both genetic and behavioral determinants [2].

Acute nonobstructive pyelonephritis is a renal infection characterized by costovertebral angle pain and tenderness, often with fever; it occurs in the same population that experiences acute uncomplicated urinary infection.

Complicated urinary tract infection, which may involve either the bladder or kidneys, is a symptomatic urinary infection in individuals with functional or structural abnormalities of the genitourinary tract [3].

Uncomplicated urinary infection occurs rarely in men, and urinary infection in men is usually considered complicated.

1. Professor and Ex-chairman, Dept. Urology, BSMMU, Dhaka

2. Consultant, Urology and Transplantation Foundation of Bangladesh

3. Professor, Department of Urology, Bangladesh Medical College

4. Consultant Urologist, Lab Aid Hospital, Dhaka

5. Ex-Professor of Urology, BSMMU, Dhaka \& Advisory Editor

6. Professor of Urology, NICRH, Dhaka \& Member, Editorial Board Correspondences: Salam MA, Urology and Transplantation Foundation of Bangladesh

Received: 9 August 2017

Accepted: 05 March 2018
Reinfection is recurrent urinary tract infection with an organism originating from outside of the urinary tract, either a new bacterial strain or a strain previously isolated that has persisted in the colonizing flora of the gut or vagina [2].

Pyuria is the presence of increased numbers of polymorpho nuclear leukocytes in the urine and is evidence of an inflammatory response in the urinary tract [4].

\section{Asymptomatic Bacteriuria}

Patients with positive urine cultures who lack symptoms of a UTI have the diagnosis of asymptomatic bacteriuria. ASBU is more common in some patient populations and the prevalence increases with advancing age. It is also associated with sexual activity in young women. Patients with impaired urinary voiding or indwelling urinary devices have a much higher prevalence of ASBU[1].

The treatment of Asymptomatic Bacteriuria (ASBU) is only indicated if the bacteriuria has an associated adverse outcome ( such as development of a symptomatic urinary tract infection, bacteremia, septicemia, or progression to chronic kidney disease, etc.).

There are only 2 clinical situations where the treatment may be indicated. Pregnant women should be screened and treated for ASBU, as they have a significantly increased risk of developing pyelonephritis as well as experiencing a premature delivery and delivering a low birth weight infant. Prior to transurethral resection of the prostate (TURP) or any other urologic procedure with a risk of mucosal bleeding, patients should be screened for bacteriuria, as it has be en associated with a major increase in the risk for post procedure bacteremia and sepsis.

Treatment of ASBU in both these situations has been demonstrated to prevent these complications. Unfortunately many patients with ASBU receive treatment which they do not benefit from and in fact are likely harmed. The unnecessary treatment of ASBU can lead to antibiotic resistance, adverse drug effects, 
C. difficile infection, and contribute unnecessary costs of medical care.

Urinary tract infection (UTI) is the most common indication for antimicrobial use in hospitals and a significant proportion of this use is inappropriate or unnecessary. The Antimicrobial Stewardship Program at the Nebraska Medical Center has developed guidelines to facilitate the evaluation and treatment of UTIs. Ordering of Urine Culture: Urine cultures should only be obtained when a significant suspicion for a UTI exists based on patient symptoms. Urine culture data should always be interpreted taking into account the results of the urinalysis and patient symptoms. In the urinalysis the presence of leukocyte esterase suggests WBC will be present while nitrites suggest that gram-negative organisms are present. Neither of these findings is diagnostic of a UTI[1,12]

\section{Indication for urine culture:}

1. When clinical feature suggest a urinary tract infection is present

2. In patients who cannot provide history (intubated, demented) and have sepsis without another source to explain it

\section{Urine Culture is Not Recommended:}

1. Change in urine color, odor, or turbidity (these are typically due to patient hydration and not indicators of infection)

2. Patient lacks symptoms of UTI

3. Automatically in workup of fever or sepsis patients who can provide a history should not have a urine culture obtained as part of fever evaluation unless symptoms suggest a UTI is present

4. Pre-operative work except in urologic surgery where mucosal bleeding is anticipated

5. When a urinary catheter is placed or changed

6. At admission as routine workup

7. After treatment of UTI to document cure

\section{Interpretation of Urine Culture:}

Bacteria are frequently noted on urinalysis and cultured from urine specimens. The presence of bacteria in the urine may indicate one of 3 conditions: 1 ) specimen contamination; 2) urinary tract infection (UTI); or 3) asymptomatic bacteriuria (ASBU).

When evaluating the clinical significance of a urine culture these conditions must each be considered and classification should be based upon history and exam findings coupled with urine findings.

Specimen contamination should always be considered as this is common, particularly in female patients. High numbers of squamous cells on the urinalysis $(>20)$ suggests contamination and results of the culture should generally be ignored.

In patients with a positive urine culture, where no contamination exists, clinicians must determine if the patient is exhibiting symptoms of a UTI.

Urinary frequency or urgency, dysuria is most commonly is due to over active bladder, excess water indulgence and also could be from recent onset of Diabetes Mellitus and antibiotics are not indicated in these situation. If urine culture showed a bacterial growth they should be classified as asymptomatic bacteriuria (ASBU) $[1,2,3,5,12]$.

Symptoms typical of a UTI are urinary frequency or urgency, dysuria, new onset hematuria, suprapubic pain, costovertebral tenderness or fever. Patients with a urinary catheter in place may have more vague symptoms such as new onset or worsening fever, chills, pelvic discomfort, acute hematuria and altered mental status with no other identifiable etiology[2,3,5].

It is also important to recognize that pyuria is not an indication for treatment. Pyuria is the presence of an increased number of polymorphonuclear leukocytes in the urine (generally $>10 \mathrm{WBC} / \mathrm{hpf}$ ) and is evidence for genitourinary tract inflammation. Pyuria can be seen in patients with catheter use, sexually transmitted diseases, renal tuberculosis, interstitial nephritis or ASBU[4]. The absence of pyuria is a strong indicator that a UTI is not present and is useful in ruling out a UTI $[1,2,4,12]$

\section{Antimicrobial Stewardship Program}

Antimicrobial resistance costs money, livelihood, and lives, and threatens to undermine the effectiveness of health delivery programs. It has been described as a threat to global stability and national security. Antimicrobial use is the key driver of resistance. This selective pressure comes from a combination of overuse... and also from misuse [13].

Through a joint effort of Pharmaceutical \& Nutrition Care, Infection Control and Epidemiology, and the Infectious Diseases Division of Internal Medicine, the Antimicrobial Stewardship Program (ASP) at Nebraska Medicine has been active since August 2004. The 
mission of the ASP is to optimize the utilization of antimicrobial agents in order to realize improved patient outcomes, a positive effect on antimicrobial resistance, and an economic benefit $[12,13]$.

Antimicrobial stewardship is defined as a rational, systematic approach to the use of antimicrobial agents in order to achieve optimal outcomes. This means using the right agent, at the correct dose, for the appropriate duration in order to cure or prevent infection, while minimizing toxicity and emergence of resistance[12,13].

Fast Facts from the Centers for Disease Control and Prevention

Antibiotic use is the most important modifiable driver of antibiotic resistance, and antibiotic-resistant infections lead to higher healthcare costs, poor health outcomes, and more toxic treatments[5].

At least $30 \%$ of antibiotic courses prescribed in the outpatient setting are unnecessary, meaning that no antibiotic is needed at all. Most of this unnecessary use is for acute respiratory conditions, such as colds, bronchitis, sore throats caused by viruses, and even some sinus and ear infections[6]

Total inappropriate antibiotic use, which includes unnecessary antibiotic use plus inappropriate antibiotic selection, dosing, and duration, may approach $50 \%$ of all outpatient antibiotic use[7,9].

Antibiotics are the most common cause of adverse drug events (ADEs) in children, accounting for 7 of the top 10 drugs leading to pediatric ADE-related emergency room (ER) visits. Antibiotics are in the top three drug classes leading to ADE-related ER visits for all ages[10].

Improving antibiotic prescribing can reduce harm. A $10 \%$ decrease in inappropriate prescribing in the community can result in a $17 \%$ reduction in Clostridium difficile infection, a severe form of diarrhea usually caused by antibiotic exposure[11].

\section{References:}

1. Rubin RH, Shapiro ED, AndrioleVT, DavisRJ, Stamm WE. Evaluation of new anti-infective drugs for the treatment of urinary tract infection, Clin Infect Dis, 1992, vol. 15 Suppl 1(pg. 216 27) Google Scholar CrossRef

2. Hooton TM, Stamm WE. Diagnosis and treatment of uncomplicated urinary tract infection, Infect Dis Clin North Am , 1997, vol. 11 (pg. 551-82) Google ScholarCrossRef PubMed
3. Nicolle LE. A practical approach to the management of complicated urinary tract infection, Drugs Aging, 2001, vol. 18 (pg. 243-54) Google Scholar CrossRef PubMed

4. Stamm WE. Measurement of pyuria and its relation to bacteriuria, Am J Med, 1983, vol. 75Suppl 1B(pg. 53-58)Google Scholar CrossRefPubMed

5. Centers for Disease Control and Prevention. Antibiotic Resistance Threats in the United States. 16 September 2013. Web. 17 July 2014.

6. Fleming-Dutra, K., et al. (2016). "Prevalence of Inappropriate Antibiotic Prescriptions Among US Ambulatory Care Visits, 2010-2011." JAMA: the Journal of the American Medical Association 315(17): 1864-1873.

7. Centers for Disease Control and Prevention (CDC). Office-related antibiotic prescribing for persons aged d" 14 years — United States, 19931994 to 2007-2008. MMWR Morb Mortal Wkly Rep. 2011;60(34):1153-6.

8. Pichichero ME. Dynamics of antibiotic prescribing for children. JAMA. June 19, 2002;287(23): 3133-5.

9. Shapiro DJ, Hicks LA, Pavia AT, Hersh AL. Antibiotic prescribing for adults in ambulatory care in the USA, 2007-09. J Antimicrob Chemother. 2014;69(1):234-40.

10. Shehab, N., et al. (2016). "US emergency department visits for outpatient adverse drug events, 2013-2014." JAMA 316(20): 2115-2125.

11. Dantes, R., et al. (2015). "Association Between Outpatient Antibiotic Prescribing Practices and Community-Associated Clostridium difficile Infection." Open forum infectious diseases 2(3): ofv113-ofv113.

12. Andrea Green Hines MD, Mark E Rupp MD, and Trevor C Van Schooneveld MD Reviewed and Approved by Antimicrobial Stewardship Subcommittee of Pharmacy and Therapeutics Committee of the Nebraska Medical Center,July 2014

13. WHO Global Strategy for Containment of Antimicrobial Resistance, 2000. 